Results

\begin{tabular}{ll}
\hline Age at removal & Range 15-21 yrs, Mean 18.5 yrs, Median 18.5 yrs \\
Length of use & Mean 10.5 months \\
Inserted Pulse & 10 \\
Identified number of reasons & 7 \\
for removal: & 11 \\
$\quad$ Unscheduled bleeding & 18 total \\
Other reasons for removal & 5 \\
Weight gain & 6 \\
Mood changes & 2 \\
Bloating & 3 \\
Headaches & 2 \\
Nausea & 13 \\
Miscellaneous & 5 \\
Received bleeding management & 0 \\
Willing to accept further bleeding & \\
management & 0 \\
Requests for replacement implant &
\end{tabular}

Discussion/conclusion Unscheduled bleeding is the most common reason for premature removal of implants, however many reported multiple reasons. All removals except one required ongoing reliable contraception, but none were willing to reinsert implant. These clients require support to continue this very effective form of contraception: future support includes: Identify who may require monitoring; Stress choices at outset; Offer bleeding management at early stage; Follow up new insertions at 6/52 via telephone support from Health Advisor or Nurse. Ongoing work will include monitoring and surveys on post TOP removals.

\section{P247 QUALITY OF LIFE AND SEXUAL FUNCTION AMONGST WOMEN WITH PERSISTENT GENITAL DISCHARGE OR DERMATOSES}

${ }^{1,2}$ Nina Vora*, ${ }^{3}$ Gary Whitlock, ${ }^{2}$ Nataliya Brima, ${ }^{1}$ Angela Robinson. ${ }^{1}$ Mortimer Marke Centre, London, UK; ${ }^{2}$ University College London, London, UK; ${ }^{3} 52$ Dean Street Clinic, London, UK

\subsection{6/sextrans-2015-052126.289}

Background Existing data on the effect of genital discharge and dermatoses on the quality of life (QoL) and sexual function (SF) in women with genital complaints are limited.

Objectives To study the impact of our specialist clinic for recurrent genital problems on QoL and SF using two validated questionnaires: dermatology life quality index (DLQI) and female sexual function index (FSFI).

Methods All women attending this specialist clinic during 2013 were invited to complete both DLQI and FSFI. Questionnaires were resent six months later or completed at follow-up attendance. Paired questionnaires were analysed using Wilcoxonsigned-rank tests.

Results We received 143 responses: 99 dermatological complaints and 44 discharge complaints. Both complaints have a detrimental effect on QoL (mean \pm SD quality of life scores $8 \cdot 4 \pm 6 \cdot 6$, moderate effect on QoL vs published general population score between 0 and 1 in validation studies). SF was also impaired (score $19.6 \pm 6.9$, vs published general population mean score $30.5 \pm 5.29$ ). 13 patients fully completed DLQI pre and post clinic intervention; there was significant improvement in DLQI scores (median pre-intervention vs post-intervention scores, interquartile range (IQR): $15(12-18)$ vs 8 (6-12),
$P=0.013)$. FSFI scores did not significantly improve (18.55 $(16.5-22.5)$ vs 18.5 (14.0-22.7), $\mathrm{P}=1.000)$.

Discussion/conclusion Both QoL and SF are impaired in many women presenting with recurrent genital complaints. Appropriate assessment and management by senior physicians can significantly improve QoL in these women supporting the role of specialist clinics. There remains significant impairment to SF, warranting research into affordable interventions.

\section{P248 SENSITIVITY OF THE AMSEL'S CRITERIA COMPARED TO THE NUGENT SCORE IN ABSENCE AND IN PRESENCE OF TRICHOMONAS VAGINALIS (TV) AND/OR CANDIDA SPP AMONG WOMEN WITH SYMPTOMATIC VAGINITIS/ VAGINOSIS}

${ }^{1}$ Lucile Belley-Montfort*, ${ }^{2}$ Joel Lebed, ${ }^{3}$ Bonnie Smith, ${ }^{3}$ Melissa Farrell, ${ }^{4}$ Jane Schwebke, ${ }^{5}$ Paul Nyirjesy, ${ }^{6}$ Thomas Davis, ${ }^{6}$ DeAnna Fuller, ${ }^{7}$ Kenneth Fife, ${ }^{8}$ Safedin Sajo Beqaj. 'BD Diagnostics, Québec, QC, Canada; ${ }^{2}$ Planned Parenthood Southeastern Pennsylvania, Philadelphia, PA, USA; ${ }^{3}$ Planned Parenthood Gulf Coast, Houston, TX, USA; ${ }^{4}$ The University of Alabama at Birmingham, Birmingham, AL, USA; ${ }^{5}$ Drexel University, Philadelphia, PA, USA; ${ }^{8}$ Pathology Inc, Torrance, CA, USA; ${ }^{6}$ Wishard Health Services, Indianapolis, IN, USA; ${ }^{7}$ Indiana University, Indianapolis, IN, USA

\subsection{6/sextrans-2015-052126.290}

Background/introduction In a multicenter clinical trial funded by $\mathrm{BD}$, we observed less accurate clinician diagnosis of bacterial vaginosis (BV) based on clinical observations when Trichomonas vaginalis (TV) and/or Candida spp. were also detected by the trial Reference Methods than when only BV was detected.

Aim(s)/objectives To determine the sensitivity of each criterion and of the overall Amsel's criteria (3/4 criteria met), the results of the Amsel's corresponding to the sub-population of specimens that gave a Nugent score of 7-10 were analysed.

Methods Following informed consent, women with symptoms of vaginitis/vaginosis were included in the trial. The four Amsel's criteria and the Nugent score were performed. Evaluation for trichomoniasis by wet mount and culture (InPouch $^{\mathrm{TM}}$ TV, Biomed) were performed. Candida colonies were isolated (BBL ${ }^{\mathrm{TM}}$ Sabouraud Dextrose Agar, Emmons and BBL ${ }^{\mathrm{TM}} \mathrm{CHROMAgar}^{\mathrm{TM}}$ Candida plate, BD) and identified by ITS-2 bi-directional sequencing (Accugenix ${ }^{\circledR}$ ).

Results In total, 269/497 (54.1\%) specimens gave a Nugent score of 7-10. Amongst them, TV and/or Candida spp. were found in 100 specimens (37.2\%). The sensitivity of clue cells, amine test, vaginal $\mathrm{pH}$, BV vaginal discharge, and overall Amsel's criteria in absence of TV and/or Candida spp. was $86.3 \%$, $82.7 \%, 91.1 \%, 71.0 \%$, and $84.6 \%$ respectively. In presence of TV and/or Candida spp., the sensitivity was $63.6 \%, 64.0 \%$, $75.0 \%, 42.0 \%$, and $60.0 \%$ respectively ( $\mathrm{p}$ values $\leq 0.0009$ for all comparisons).

Discussion/conclusion The sensitivity of the Amsel's criteria in women with BV decreases when TV and/or Candida spp. are present. The BV vaginal discharge is the least sensitive criterion.

\section{P249 SO WHAT DO WOMEN WANT - ESTABLISHING A WOMEN'S SEXUAL HEALTH SERVICE}

Emma Collins*, Michelle Hawkins. BSUH NHS Trust, Brighton, UK

\subsection{6/sextrans-2015-052126.291}

Introduction Patient and public feedback has highlighted the need for targeted sexual health services for women in our city. 